\title{
One-qubit reduced states of a pure many-qubit state: polygon inequalities
}

\author{
A. Higuchi, ${ }^{1}$ A. Sudbery ${ }^{2}$ and J. Szulc ${ }^{3}$ \\ Department of Mathematics, University of York, Heslington, York YO10 5DD, UK \\ email:1'ah28@york.ac.uk, ${ }^{2}$ as2@york.ac.uk, ${ }^{3} j s 115 @ y o r k . a c . u k$
}

(Dated: 15 August 2002)

\begin{abstract}
We show that a necessary and sufficient condition for a set of $n$ one-qubit mixed states to be the reduced states of a pure $n$-qubit state is that their smaller eigenvalues should satisfy polygon inequalities: each of them must be no greater than the sum of the others.
\end{abstract}

PACS numbers: PACS numbers: 03.67.-a, 03.65.Ta, 03.65.Ud

In this paper we study the quantum analogue of the marginal distributions of a joint probability distribution. The results reveal some surprising aspects of pure states of multipartite quantum systems.

In a pure state of many particles, each subset $P$ of the particles is in a mixed state $\rho_{P}$, the reduced state of the subset $P$. These reduced states are subject to conditions such as the consistency conditions

$$
\operatorname{tr}_{Q} \rho_{P \cup Q}=\operatorname{tr}_{R} \rho_{P \cup R}
$$

for any subsets $P, Q, R$ such that $P$ and $Q$ are disjoint and $P$ and $R$ are disjoint. However, equations of this type do not exhaust the conditions: Linden, Popescu and Wootters [1] have given an example of a set of three twoqubit density matrices which satisfy all such conditions but cannot be the reduced two-qubit states of a pure state of three qubits. Another example is given by the system of four qubits, in which it is impossible for all three reduced two-qubit states to be totally mixed [2] though all the consistency conditions (11) are satisfied. The full set of conditions is not known.

In this paper we completely determine the possible one-qubit reduced states of a pure state of $n$ qubits. (There is no problem for mixed states, since any $n$ onequbit mixed states $\rho_{1}, \ldots \rho_{n}$ are the reduced states of the $n$-qubit mixed state $\rho_{1} \otimes \cdots \otimes \rho_{n}$.) This is equivalent to determining the set of possible eigenvalues for each qubit, i.e. the marginal probability distributions. Brun and Cohen [3] have pointed out that in the case $n=3$ the determinants of the reduced states must satisfy triangle inequalities, but not every set of three $2 \times 2$ density matrices satisfying these inequalities is a possible set of reduced states of a pure three-qubit state. We will show that such density matrices must satisfy the stronger condition that their smaller eigenvalues satisfy triangle inequalities. For $n$ qubits, a necessary condition is the obvious generalisation of the triangle inequalities:

$$
\lambda_{k} \leq \lambda_{1}+\cdots+\lambda_{k-1}+\lambda_{k+1}+\cdots+\lambda_{n}
$$

where the eigenvalues of the density matrix $\rho_{k}$ of the $k$ th qubit are $\lambda_{k}$ and $1-\lambda_{k}$ with $\lambda_{k} \leq 1 / 2$. These inequalities completely characterise the possible sets of eigenvalues, and therefore the possible reduced one-qubit states, of a pure $n$-qubit state.
These results reveal some surprising aspects of pure quantum states. One concerns the comparison with the classical notion of a pure state in the sense of a probability distribution. The notion of a pure state applies to both quantum and classical systems, if we consider a state of a classical system to be a probability distribution for the values of variables which actually have precise values. Then a pure state of a classical system (defined to be an extreme point of the convex set of probability distributions) corresponds to perfect knowledge of the variables, so that all probabilities are 0 or 1 . A pure state of a quantum system corresponds to maximum knowledge, but the characteristic feature of quantum mechanics is that even in a pure state there are variables for which perfect knowledge is not available; indeed, any possible probability distribution will occur for some variable. However, given any pure state there is always some physical variable for which the probabilities are all 0 or 1 (for example, the projection operator onto the state). This is the quantum version of the above characterisation of classical pure states.

This analogy between quantum and classical pure states disappears when we consider marginal states. Classically, a joint distribution of many variables is pure if and only if each one-variable marginal distribution is pure. We know that this is not true in quantum mechanics; the fact that a pure multipartite state may give mixed one-party states is the phenomenon of entanglement. Nevertheless, our results place limits on the oneparty states; not every probability distribution is possible.

The most surprising aspect of this is that it is a statement about an overall property of the whole system, namely the purity of its state, which depends only on local measurements. We emphasize that this is only a negative statement: from the knowledge that the oneparty reduced states satisfy the polygon inequalities one cannot normally deduce that the multipartite state must be pure (this is only possible if the one-party states are themselves pure); but the local information in a violation of the polynomial inequalities does give the overall information that the multipartite state cannot be pure.

To prove the assertion (2), we expand the pure $n$-qubit state $|\Psi\rangle$ in the Schmidt basis $\left[3,4,[5]\right.$. Let $\left|\phi_{0}^{(k)}\right\rangle$ and 
$\left|\phi_{1}^{(k)}\right\rangle$ be the eigenstates of the reduced state $\rho_{k}$ with eigenvalues $\lambda_{k}$ and $1-\lambda_{k}$ respectively; we will show that

$$
\lambda_{1} \leq \lambda_{2}+\cdots+\lambda_{n} .
$$

We assume $\lambda_{1}>0$ (if not, the inequality (3) is trivially satisfied). We note that $\left|\phi_{0}^{(1)}\right\rangle$ and $\left|\phi_{1}^{(1)}\right\rangle$ are the states of the first qubit occurring in the Schmidt decomposition of $|\Psi\rangle$ when the system is divided into two parts, one containing the first qubit and the other containing the rest, so

$$
|\Psi\rangle=A\left|\phi_{0}^{(1)}\right\rangle\left|\Phi_{0}^{(1)}\right\rangle+B\left|\phi_{1}^{(1)}\right\rangle\left|\Phi_{1}^{(1)}\right\rangle
$$

where $\left|\Phi_{0}^{(1)}\right\rangle$ and $\left|\Phi_{1}^{(1)}\right\rangle$ are orthogonal normalised $(n-1)$ qubit states, $A$ and $B$ are real and $A^{2}=\lambda_{1}=1-B^{2}$. Since $\lambda_{1}$ is the smaller eigenvalue, $0<A \leq B$. We write $\left|i_{1} \ldots i_{n}\right\rangle=\left|\phi_{i_{1}}^{(1)}\right\rangle \ldots\left|\phi_{i_{n}}^{(n)}\right\rangle$ and expand $|\Psi\rangle$ in this basis:

$$
\begin{aligned}
|\Psi\rangle= & A \sum_{i_{2}, i_{3}, \ldots, i_{n}} a_{i_{2} i_{3} \cdots i_{n}}\left|0 i_{2} i_{3} \cdots i_{n}\right\rangle \\
& +B \sum_{i_{2}, i_{3}, \ldots, i_{n}} b_{i_{2} i_{3} \cdots i_{n}}\left|1 i_{2} i_{3} \cdots i_{n}\right\rangle
\end{aligned}
$$

with

$$
\sum_{i_{2}, i_{3}, \ldots, i_{n}}\left|a_{i_{2} i_{3} \cdots i_{n}}\right|^{2}=\sum_{i_{2}, i_{3}, \ldots, i_{n}}\left|b_{i_{2} i_{3} \cdots i_{n}}\right|^{2}=1 .
$$

Then

$$
\begin{aligned}
\lambda_{k}= & A^{2} \sum_{i_{2}, i_{3}, \ldots, i_{n} ; i_{k}=0}\left|a_{i_{2} i_{3} \cdots i_{n}}\right|^{2} \\
& +B^{2} \sum_{i_{2}, i_{3}, \ldots, i_{n} ; i_{k}=0}\left|b_{i_{2} i_{3} \cdots i_{n}}\right|^{2}
\end{aligned}
$$

so that

$$
\begin{aligned}
\lambda_{2}+\cdots+\lambda_{n}= & A^{2} \sum_{i_{2}, i_{3}, \ldots, i_{n}} N_{i_{2} i_{3} \cdots i_{n}}\left|a_{i_{2} i_{3} \cdots i_{n}}\right|^{2} \\
& +B^{2} \sum_{i_{2}, i_{3}, \ldots, i_{n}} N_{i_{2} i_{3} \cdots i_{n}}\left|b_{i_{2} i_{3} \cdots i_{n}}\right|^{2}
\end{aligned}
$$

where

$$
N_{i_{2} i_{3} \cdots i_{n}} \equiv n-1-\left(i_{2}+i_{3}+\cdots+i_{n}\right)
$$

is the number of times " 0 " appears in $\left(i_{2}, i_{3}, \ldots, i_{n}\right)$. Hence $N_{i_{2} i_{3} \cdots i_{n}} \geq 1$ if $\left(i_{2}, i_{3}, \ldots, i_{n}\right) \neq(1,1, \ldots, 1)$. This implies that

$$
\begin{aligned}
\lambda_{2}+\cdots+\lambda_{n} \geq & A^{2}\left(\sum_{i_{2}, i_{3}, \ldots, i_{n}}\left|a_{i_{2} i_{3} \cdots i_{n}}\right|^{2}-\left|a_{11 \cdots 1}\right|^{2}\right) \\
& +B^{2}\left(\sum_{i_{2}, i_{3}, \ldots, i_{n}}\left|b_{i_{2} i_{3} \cdots i_{n}}\right|^{2}-\left|b_{11 \cdots 1}\right|^{2}\right) \\
= & A^{2}\left(1-\left|a_{11 \cdots 1}\right|^{2}\right)+B^{2}\left(1-\left|b_{11 \cdots 1}\right|^{2}\right) \\
\geq & A^{2}\left(2-\left|a_{11 \cdots 1}\right|^{2}-\left|b_{11 \cdots 1}\right|^{2}\right),
\end{aligned}
$$

since $B^{2} \geq A^{2}$. Now by the orthogonality of $\left|\Phi_{0}^{(1)}\right\rangle$ and $\left|\Phi_{1}^{(1)}\right\rangle$ in (4),

$$
\sum_{i_{2}, i_{3}, \ldots, i_{n}} a_{i_{2} i_{3} \cdots i_{n}}{ }^{*} b_{i_{2} i_{3} \cdots i_{n}}=0,
$$

so, using the Cauchy-Schwarz inequality,

$$
\begin{aligned}
\left|a_{11 \cdots 1}\right|^{2}\left|b_{11 \cdots 1}\right|^{2} \leq & \left(\sum_{i_{2}, i_{3}, \ldots, i_{n}}\left|a_{i_{2} i_{3} \cdots i_{n}}\right|^{2}-\left|a_{11 \cdots 1}\right|^{2}\right) \\
& \left(\sum_{i_{2}, i_{3}, \ldots, i_{n}}\left|b_{i_{2} i_{3} \cdots i_{n}}\right|^{2}-\left|b_{11 \cdots 1}\right|^{2}\right) \\
= & \left(1-\left|a_{11 \cdots 1}\right|^{2}\right)\left(1-\left|b_{11 \cdots 1}\right|^{2}\right) .
\end{aligned}
$$

Therefore

$$
\left|a_{11 \cdots 1}\right|^{2}+\left|b_{11 \cdots 1}\right|^{2} \leq 1 .
$$

Hence from Eq. (7) we have

$$
\lambda_{2}+\lambda_{3}+\cdots+\lambda_{n} \geq A^{2}=\lambda_{1} .
$$

Clearly there are similar inequalities with each of the $\lambda_{k}$ on the right-hand side. We call these the polygon inequalities.

To show that these inequalities define exactly the set of possible one-qubit reduced states, we prove that given any real numbers $\left\{\lambda_{1}, \ldots, \lambda_{n}\right\}$ lying between 0 and $1 / 2$ and satisfying (2) we can find an $n$-qubit state for which $\lambda_{k}$ is the smaller eigenvalue of the reduced density matrix $\rho_{k}$. We will suppose that $\lambda_{1}$ is the largest of the numbers, so that

$$
0 \leq \lambda_{i} \leq \lambda_{1} \leq 1 / 2, \quad i=2,3, \ldots n
$$

For $n=3$ let

$$
\left|\Psi_{3}\right\rangle \equiv a|100\rangle+b|010\rangle+c|001\rangle+d|111\rangle,
$$

where $a, b, c$ and $d$ are real and satisfy $a^{2}+b^{2}+c^{2}+d^{2}=1$. Then for each of the three qubits the eigenvectors of the one-qubit reduced state are $|0\rangle$ and $|1\rangle$. Let $\lambda_{i}$ be the eigenvalue corresponding to $|0\rangle$ for the $i$ th qubit. Then

$$
\begin{aligned}
& \lambda_{1}=b^{2}+c^{2}, \\
& \lambda_{2}=c^{2}+a^{2}, \\
& \lambda_{3}=a^{2}+b^{2} .
\end{aligned}
$$

These can be solved as

$$
\begin{aligned}
& a^{2}=\frac{1}{2}\left(\lambda_{2}+\lambda_{3}-\lambda_{1}\right), \\
& b^{2}=\frac{1}{2}\left(\lambda_{3}+\lambda_{1}-\lambda_{2}\right), \\
& c^{2}=\frac{1}{2}\left(\lambda_{1}+\lambda_{2}-\lambda_{3}\right) .
\end{aligned}
$$


Thus if the $\lambda_{i}$ satisfy the triangle inequalities there is a real solution $(a, b, c)$ of the equations, and if $\lambda_{i} \leq 1 / 2$ this solution satisfies

$$
a^{2}+b^{2}+c^{2}=\frac{\lambda_{1}+\lambda_{2}+\lambda_{3}}{2} \leq 3 / 4,
$$

so that $a^{2}+b^{2}+c^{2} \leq 1$ and it is possible to find a normalised state $|\Psi\rangle$. Hence, there is a state with arbitrary eigenvalues satisfying Eqs. (3) and (12) for $n=3$.

Now assume that this is true for any $(n-1)$-qubit system. Suppose that $\lambda_{n}$ is the smallest of the $\lambda_{i}$, and define $\Lambda_{1} \equiv \lambda_{1}-\lambda_{n}$. There are two cases to consider. If $\Lambda_{1} \geq \lambda_{i}$ for $2 \leq i \leq n-1$, then $\left(\Lambda_{1}, \lambda_{2}, \ldots, \lambda_{n-1}\right)$ satisfies Eqs. (3) and (12) with $n$ replaced by $n-1$ and $\lambda_{1}$ by $\Lambda_{1}$. In the second case, $\Lambda_{1}<\lambda_{m}$ for some $m$, say $m=2$; we can suppose that $\lambda_{2} \geq \lambda_{i}$ for $i=3, \ldots, n-1$. Then

$$
\begin{aligned}
\Lambda_{1}+\lambda_{3}+\cdots+\lambda_{n-1} & =\lambda_{1}+\left(\lambda_{3}-\lambda_{n}\right)+\lambda_{4}+\cdots+\lambda_{n-1} \\
& \geq \lambda_{1} \geq \lambda_{2} .
\end{aligned}
$$

Hence the set $\left(\lambda_{2}, \Lambda_{1}, \lambda_{3}, \ldots, \lambda_{n-1}\right)$ satisfies Eqs. (3) and (12) with $n$ replaced by $n-1$ and $\lambda_{1}$ and $\lambda_{2}$ replaced by $\lambda_{2}$ and $\Lambda_{1}$, respectively. In either case there is a state for which $\Lambda_{1}, \lambda_{2}, \ldots, \lambda_{n-1}$ are the smaller eigenvalues of the reduced density matrices. Let this state be

$$
\left|\Psi_{n-1}\right\rangle=|0\rangle|\phi\rangle+|1\rangle|\psi\rangle,
$$

where $|\phi\rangle$ and $|\psi\rangle$ are $(n-2)$-qubit states satisfying $\langle\phi \mid \phi\rangle=\Lambda_{1},\langle\psi \mid \psi\rangle=1-\Lambda_{1}$ and $\langle\phi \mid \psi\rangle=0$, and $\lambda_{2}$, $\lambda_{3}, \ldots, \lambda_{n-1}$ are the smaller eigenvalues of the one-qubit reduced density matrices of $|\phi\rangle\langle\phi|+| \psi\rangle\langle\psi|$. Now consider the following $n$-qubit state:

$$
\left|\Psi_{n}\right\rangle=|0\rangle|\phi\rangle|1\rangle+\sin \chi|0\rangle|\psi\rangle|0\rangle+\cos \chi|1\rangle|\psi\rangle|1\rangle .
$$

The smaller eigenvalue $\tilde{\lambda}_{i}$ of this state for the $i$ th qubit with $2 \leq i \leq n-1$ is again the smaller eigenvalue of the $i$ th one-qubit reduced density matrix of $|\phi\rangle\langle\phi|+| \psi\rangle\langle\psi|$. Hence $\tilde{\lambda}_{i}=\lambda_{i}$ for $2 \leq i \leq n-1$. The $n$th and 1 st eigenvalues corresponding to the eigenvectors $|0\rangle$ are

$$
\begin{aligned}
& \tilde{\lambda}_{n}=\sin ^{2} \chi\langle\psi \mid \psi\rangle, \\
& \tilde{\lambda}_{1}=\langle\phi \mid \phi\rangle+\sin ^{2} \chi\langle\psi \mid \psi\rangle=\Lambda_{1}+\tilde{\lambda}_{n} .
\end{aligned}
$$

Since $\langle\psi \mid \psi\rangle \geq 1 / 2$, one can choose $\chi$ so that $\tilde{\lambda}_{n}=\lambda_{n}$. Then (24) gives $\tilde{\lambda}_{1}=\lambda_{1}$.
We conclude by induction that a state with the required one-qubit eigenvalues exists for any $n$.

Of course, it does not follow that if an $n$-qubit state has one-qubit reduced states whose eigenvalues satisfy the polygon inequalities, then the $n$-qubit state must be pure. In general, a given set of one-qubit reduced states can be obtained from many different $n$-qubit states, most of which will be mixed: for example, the tensor product of the one-qubit states, which will be mixed if any of the one-qubit states are mixed. It is only when all the onequbit states are pure that the $n$-qubit state from which they arise must be pure.

Purity of the $n$-qubit state places no restriction on the eigenstates of the one-qubit reduced states, since any pair of orthogonal eigenstates can be transformed to any other by local unitary operations. The complete set of one-qubit reduced states which can arise from a pure $n$ qubit state is therefore determined by the set of possible eigenvalues that we have described. The situation for the reduced states of larger subsets is likely to be more complicated. The example of two-qubit subsets of a system of four qubits shows that polygon inequalities (3) for any combination of eigenvalues are not sufficient, for these would allow all reduced states to be totally mixed (all eigenvalues equal to $1 / 4$ ), which is not possible 22]. It would be interesting to know the exact set of marginal two-qubit probabilities for this system.

The situation for qudits (particles whose state spaces have dimension $d$ ) also appears to be more complicated. An argument similar to the above shows that the eigenvalues of the one-qudit reduced states must satisfy (3) with each $\lambda_{i}$ replaced by the sum of all but the largest eigenvalue of qudit $i$ (so the largest eigenvalues satisfy the same inequality as for qubits), but there appear to be further inequalities that must be satisfied. This is under investigation.

[1] N. Linden, S. Popescu and W. K. Wootters, quant-ph/ 0207109

[2] A. Higuchi and A. Sudbery, Phys. Lett. A 273, 213 (2000).

[3] T. A. Brun and O. Cohen, Phys. Lett. A 281, 88 (2001).

[4] O. Cohen and T. A. Brun, Phys. Rev. Lett. 84, 5908 (2000).

[5] A. Sudbery, J. Phys. A 34, 643 (2001) 